- Original Article

\title{
Comparison of Medical Students' Satisfaction with Family Medicine Clerkships between University Hospitals and Community Hospitals or Clinics
}

\author{
Eal Whan Park* \\ Department of Family Medicine, Dankook University College of Medicine, Cheonan, Korea
}

Background: The purpose of this study was to compare students' awareness of and satisfaction with clerkships in family medicine between a university hospital and a community hospital or clinic.

Methods: Thirty-eight 4th year medical students who were undergoing a clerkship in family medicine in the 1st semester of 2012 were surveyed via questionnaire. The questionnaire was administered both before and after the clerkship.

Results: External clerkships were completed in eight family medicine clinics and two regional hospitals. At preclerkship, participants showed strong expectation for understanding primary care and recognition of the need for community clerkship, mean scores of $4.3 \pm 0.5$ and $4.1 \pm 0.7$, respectively. At post-clerkship, participants showed a significant increase in recognition of the need for community clerkship $(4.7 \pm 0.5, \mathrm{P}<0.001)$. The pre-clerkship recognition of differences in patient characteristics between university hospitals and community hospitals or clinics was $4.1 \pm 0.7$; at post-clerkship, it was $3.9 \pm 0.7$. Students' confidence in their ability to see a first-visit patient and their expectation of improved interviewing skills both significantly increased at post-clerkship $(\mathrm{P}<0.01)$. Satisfaction with feedback from preceptors and overall satisfaction with the clerkship also significantly increased, but only for the university hospital clerkship $(\mathrm{P}<0.01)$.

Conclusion: Students' post-clerkship satisfaction was uniformly high for both clerkships. At pre-clerkship, students were aware of the differences in patient characteristics between university hospitals and community hospitals or clinics, and this awareness did not change by the end of the clerkship.

Keywords: Medical Students; Clinical Clerkship; Primary Health Care; Community Medicine 


\section{INTRODUCTION}

Effective teaching of family medicine to medical students requires not only lecturing on core knowledge but also the use of clinical clerkships. These clerkships can help students comprehensively understand the characteristics of family practice and of how to approach general problems encountered in primary care.

The required learning outcomes of clinical clerkships are as follows: the role of family medicine in primary care, holistic management of common health problems, family-centered and community-oriented primary care, diagnosis and treatment of patients with common problems in primary care, interview skills, effective patient education, knowledge and application of problem-oriented medical records, and understanding and application of the concepts of health promotion and disease prevention. ${ }^{1)}$

Community-oriented primary care is considered an important aspect of clerkships, as it helps medical students bridge the gap between academic medicine and the community that medicine aims to serve. Furthermore, it can teach medical students to adopt a community-responsive attitude and a public health perspective, and to understand chronic disease management, patient-centeredness, and healthcare systems. $^{2,3)}$

According to the results of a 2004 survey, ${ }^{1)}$ family medicine clerkships existed within 27 of the 41 universities canvassed. In 15 of those 27 universities, the clerkship was mandatory, while in 21 of them, a community clerkship was included as part of the overall family medicine clerkship. In most of those universities, the community clerkships were carried out in a family medicine clinic over the course of half a day to two days.

Later, a 2013 survey ${ }^{4)}$ of 22 universities revealed that all of the studied institutions had implemented a family medicine clerkship. Furthermore, in 15 (68\%) of those universities, the clerkship was mandatory. The duration of the clerkship was one week in 12 of the universities $(60 \%)$ and two weeks in four of the universities (20\%). Most of the medical colleges offering clinical clerkships in family medicine have made an effort to install them in primary or secondary health care settings, with, crucially, the cooperation of university hospitals. However, according to the results of a survey of medical students, most clerkships in family medicine were community clerkships taking place in family physicians' clinics, and lasted for approximately half a day to two days.

Questions have been raised regarding the uniqueness and effectiveness of community clerkships for primary care compared with those provided in university hospitals, ${ }^{5}$ and several studies have compared medical students' learning experiences between academic and community practices in family medicine ${ }^{6-9)}$ and other specialties. ${ }^{10,11)}$ However, thus far, there has been no such comparisons in South Korea.

The purpose of this study was to determine the differences in 4th year medical students' expectations regarding, awareness of, and satisfaction with elective family medicine clerkships between a university hospital and off-campus community hospitals or clinics.

\section{METHODS}

\section{Subjects and Measures}

From March 19 to June 22, 2012, numerous 4th year medical students completed an elective clerkship within the department of family medicine. Students served out their four-day clerkship in a university hospital (1st to 3rd day, and 5th day), and they completed a daylong community clerkship in a family physician's clinic or the family medicine department of a community hospital (4th day). Just prior to the start of the clerkship, we determined students' expectations of each clerkship; then, immediately upon completion of the clerkships, we examined differences in content and students' experiences between the clerkship sites, as well as their awareness of and satisfaction with each clerkship.

In the pre-clerkship questionnaire, five items were devoted to the students' expectations of the family medicine clerkship. These items were all on a 5-point scale (1-5 points). Besides these items, we included a list of 31 common clinical problems or diseases that were expected to be encountered during the clerkship, along with 24 common educational experiences. Students were asked to indicate each of the problems, diseases, and experiences that they expected to encounter during the clerkship. The students also were asked to indicate any other anticipated clinical problems or diseases and educational experiences. The pre-clerkship questionnaire was completed on the first day of the clerkship (i.e., orientation).

The post-clerkship questionnaire comprised two parts, one concerning the community hospital or clinic and the other concerning the university hospital. The community clerkship part contained ten items, all of which were evaluated on a 5-point scale (1-5 points), investigating students' recognition of and satisfaction with the clerkship. Furthermore, students were again asked to select, from among the 31 clinical problems or diseases and 24 educational experiences listed on the pre-clerkship questionnaire, those actually encountered during the clerkship (and to indicate any others they encountered not on the respective lists). Additionally, the students were asked to freely describe what they wanted to experience through the community clerkship, the positive educational experiences they obtained as a result of participating, and what should be changed to improve the clerkship.

The contents of the university hospital part of the questionnaire were the same as those in the community clerkship part. The postclerkship questionnaire was completed just before the professor gave feedback on the clerkship at the end of the rotation.

\section{Statistical Analysis}

Frequency analyses and paired t-tests were performed to compare changes in mean values of the continuous variables between pre- and post-clerkship. IBM SPSS Statistics ver. 21.0 (IBM Co., Armonk, NY, USA) was used for all statistical analyses. 
Table 1. Comparison of students' pre-clerkship expectations and post-clerkship experiences

\begin{tabular}{|c|c|c|c|}
\hline Items & Pre-clerkship & Post-clerkship & P-value \\
\hline Expectation of understanding primary care & $4.3 \pm 0.5$ & $4.2 \pm 0.6$ & 0.26 \\
\hline Recognition of the need of clerkships in community hospitals or clinics & $4.1 \pm 0.7$ & $4.7 \pm 0.5$ & $<0.001$ \\
\hline Recognition of the difference in patient characteristics between a community hospital or clinic and a university hospital & $4.1 \pm 0.7$ & $3.9 \pm 0.7$ & 0.32 \\
\hline Confidence in ability to interview a first-visit patient & $2.7 \pm 0.7$ & $4.2 \pm 0.6$ & $<0.001$ \\
\hline Expectation of improved interviewing skills via video recording and professors' feedback & $4.3 \pm 0.6$ & $4.7 \pm 0.5$ & 0.006 \\
\hline
\end{tabular}

Values are presented as mean \pm standard deviation.

*From paired t-test for comparing the differences between two means.

Table 2. Diseases expected to be encountered or actually experienced by students in family medicine clerkships $(\mathrm{N}=38)$

\begin{tabular}{|c|c|}
\hline Variable & Value \\
\hline \multicolumn{2}{|c|}{ Diseases expected to be encountered in pre-clerkship } \\
\hline Hypertension & $36(94.7)$ \\
\hline Upper respiratory infection & $32(84.2)$ \\
\hline Diabetes mellitus & $31(81.6)$ \\
\hline Peptic ulcer disease & $28(73.7)$ \\
\hline Depression & $26(68.4)$ \\
\hline Obesity & $25(65.8)$ \\
\hline Functional gastrointestinal disorder & $25(65.8)$ \\
\hline Vaccination & $24(63.2)$ \\
\hline Migraine & $23(60.5)$ \\
\hline Atopic dermatitis & $23(60.5)$ \\
\hline \multicolumn{2}{|c|}{ Diseases experienced in community hospital or clinic clerkship } \\
\hline Hypertension & $37(97.4)$ \\
\hline Diabetes mellitus & $36(94.7)$ \\
\hline Upper respiratory infection & $32(84.2)$ \\
\hline Back pain & $21(55.3)$ \\
\hline Functional gastrointestinal disorder & $20(52.6)$ \\
\hline Allergic rhinitis & $19(50.0)$ \\
\hline Vaccination & $16(42.1)$ \\
\hline Peptic ulcer disease & $12(31.6)$ \\
\hline Obesity & $12(31.6)$ \\
\hline Osteoarthritis & $12(31.6)$ \\
\hline \multicolumn{2}{|c|}{ Diseases experienced in university hospital clerkship } \\
\hline Diabetes mellitus & $38(100.0)$ \\
\hline Hypertension & $36(94.7)$ \\
\hline Upper respiratory infection & $34(89.5)$ \\
\hline Peptic ulcer disease & $20(52.6)$ \\
\hline Functional gastrointestinal disorder & $19(50.0)$ \\
\hline Tension headache & $18(47.4)$ \\
\hline Migraine & $18(47.4)$ \\
\hline Depression & $18(47.4)$ \\
\hline Obesity & $17(44.7)$ \\
\hline Anxiety & $15(39.5)$ \\
\hline
\end{tabular}

Values are presented as number (\%).

\section{RESULTS}

\section{Demographic Characteristics of Study Subjects}

Thirty-eight 4th year students from among 12 small groups who elected to complete a clerkship in family medicine were surveyed. The mean age \pm standard deviation (SD) of the students was $25.6 \pm 1.7$ years old (range, 23-33 years old). Thirty-one of the students were male (81.6\%), and seven were female (18.4\%). Thirty students (78.9\%) com- pleted their community clerkship in one of eight clinics (six in Cheonan, one in Asan, and one in Hongseong), four (10.5\%) in the family medicine department of a community public hospital, and four (10.5\%) in the family medicine department of a community Orientalmedicine hospital. All of the community preceptors were appointed to professors specializing in outpatient care in the university hospital. The distance from the university hospital to the community clinic or hospital was an average of 30 minutes to one hour by car. Each week, one student was assigned to each clerkship site; over the course of the semester, a total of two to four students were assigned to each site.

\section{Students' Pre-clerkship Expectations for Family Medicine Clerkships}

In the pre-clerkship survey results, students obtained a mean $\pm S D$ of $4.3 \pm 0.5$ for expectation regarding how the clerkship would help with their understanding of primary care. Furthermore, they scored $4.1 \pm 0.7$ on the item concerning recognition of the need for a community clerkship in a community hospital or clinic, and on the item concerning awareness of the differences in patient characteristics between a university hospital and a community hospital or clinic. Students had a mean score of $2.7 \pm 0.7$ for self-confidence in their ability to interview a patient visiting an outpatient clinic for the first time, and $4.3 \pm 0.6$ for expectation for improved interviewing skills through professor feedback using video-recorded interviews (Table 1).

Regarding the diseases expected to be experienced during the clerkship, the most common ones listed were as follows: hypertension (94.7\%), upper respiratory infection (84.2\%), diabetes mellitus (81.6\%), peptic ulcer disease (73.7\%), and depression (68.4\%) (Table 2).

The skills or educational experiences that students most commonly expected to encounter in the clerkship were smoking cessation counseling (86.8\%), communication and interview skills (81.6\%), observation of outpatient clinic (81.6\%), diagnosis and treatment of common diseases (81.6\%), and obesity management (76.3\%), among others (Table 3).

\section{Students' Post-clerkship Awareness of and Satisfaction with the Family Medicine Clerkship in a Community Hospital or Clinic}

In the post-clerkship survey results, the diseases experienced most frequently by students in the community hospital or clinic were hypertension (97.4\%), diabetes (94.7\%), upper respiratory infection (84.2\%), 
back pain (55.3\%), and functional gastrointestinal disorder (52.6\%) (Table 2). The skills or educational experiences obtained or encountered most frequently by the students were communication and interview skills (100.0\%), observation of outpatient clinics (100.0\%), diagnosis and treatment of common diseases (94.7\%), evidence-based

Table 3. The educational experiences expected to be encountered or experienced in family medicine clerkships $(\mathrm{N}=38)$

\begin{tabular}{ll}
\hline \multicolumn{1}{c}{ Variable } & Value \\
\hline Educational experiences expected to be encountered at pre-clerkship \\
Counseling for smoking cessation & $33(86.8)$ \\
Communication skills & $31(81.6)$ \\
Observation in ambulatory clinics & $31(81.6)$ \\
Diagnosis and management of common diseases & $31(81.6)$ \\
Counseling for obesity & $29(76.3)$ \\
Counseling for problem drinking & $29(76.3)$ \\
Chronic fatigue care & $28(73.7)$ \\
Understanding primary care & $25(65.8)$ \\
Climacteric changes & $20(52.6)$ \\
Lifelong health maintenance & $19(50.0)$ \\
Educational experiences in community hospital or clinic clerkship & \\
Communication skills & $38(100.0)$ \\
Observation in an outpatient clinic & $38(100.0)$ \\
Diagnosis and management of common diseases & $36(94.7)$ \\
Understanding primary care & $35(92.1)$ \\
Introduction rounding in outpatient clinic & $32(84.2)$ \\
Evidence-based prescription & $18(47.4)$ \\
Geriatric care & $17(44.7)$ \\
Lifelong health maintenance & $16(42.1)$ \\
Clerkship of clinical procedures & $15(39.5)$ \\
Chronic fatigue care & $15(39.5)$ \\
Educational experiences in university hospital clerkship & \\
Communication skills & $38(100.0)$ \\
Diagnosis and management of common diseases & $38(100.0)$ \\
Observation in an outpatient clinic & $37(97.4)$ \\
Understanding primary care & $36(94.7)$ \\
Use of genogram and family resources & $35(92.1)$ \\
Counseling for smoking cessation & $34(89.5)$ \\
Introduction rounding in outpatient clinic & $32(84.2)$ \\
Participation in journal club & $23(60.5)$ \\
Lifelong health maintenance & $22(57.9)$ \\
Chronic fatigue care & $21(55.3)$ \\
Var &
\end{tabular}

Values are presented as number (\%). prescription (47.4\%), and geriatric medicine (44.7\%) (Table 3).

Students had a mean score of $4.3 \pm 0.5$ on the item concerning their understanding of the objectives of the clerkship in the community hospital or clinic, and a score of $4.4 \pm 0.8$ for the item concerning their learning the principles of primary health care through participation in the community clerkship. Furthermore, they had mean scores of $4.7 \pm 0.5$ and $3.3 \pm 1.0$ for items concerning the recognition of the need for community clerkships and understanding of the evaluation criteria of community clerkships, respectively (Table 4).

Regarding the students' satisfaction, the mean scores of the items were as follows: attitude of clerkship preceptors, $4.7 \pm 0.6$; educational environment for clerkship in a community hospital or clinic, $4.4 \pm 0.8$; clerkship evaluation process, $4.2 \pm 0.7$; feedback from clerkship preceptors, $3.5 \pm 1.1$; and clinical clerkship in a community hospital or clinic (overall satisfaction), $4.3 \pm 0.5$ (Table 4 ).

\section{Students' Post-clerkship Awareness of and Satisfaction with the Family Medicine Clerkship in the University Hospital}

The diseases or problems experienced most frequently by students while in the university hospital were diabetes (100.0\%), hypertension (97.4\%), upper respiratory infection (89.5\%), and functional gastrointestinal disorder (50.0\%) (Table 2). Furthermore, the skills or educational experiences students most frequently obtained or encountered were communication and interview skills (100.0\%), diagnosis and treatment of common diseases (100.0\%), observation in an outpatient clinic (97.4\%), skills related to utilizing genograms and family resources (92.1\%), counseling for smoking cessation (89.5\%), introduction rounding in outpatient clinics (84.2\%), participation in journal club (60.5\%), lifelong health maintenance programs (57.9\%), chronic fatigue care (55.3\%), counseling for problem drinking (52.6\%), writing problem-oriented medical records (50.0\%), and exercise prescription (50.0\%) (Table 3).

The mean score for the item concerning students' understanding of the objectives of the clerkship in the university hospital was $4.2 \pm 0.5$, while that for the item concerning their understanding of the principles of primary health care through the clerkship was $4.2 \pm 0.6$. The mean score for the item concerning students' recognition of the need

Table 4. Comparison of students' awareness of and satisfaction with family medicine clerkship between community hospitals or clinics and the university hospital

\begin{tabular}{|c|c|c|c|}
\hline Items & Community hospital or clinic & University hospital & P-value* \\
\hline Understanding of objectives of clerkship & $4.3 \pm 0.5$ & $4.2 \pm 0.5$ & 0.10 \\
\hline Understanding of principles of primary care & $4.4 \pm 0.8$ & $4.2 \pm 0.6$ & 0.12 \\
\hline Understanding of evaluation criteria of the clerkship & $3.3 \pm 1.0$ & $3.6 \pm 1.0$ & 0.14 \\
\hline Satisfaction with attitude of clerkship preceptors & $4.7 \pm 0.6$ & $4.8 \pm 0.5$ & 0.45 \\
\hline Satisfaction with educational environment of clerkship & $4.4 \pm 0.8$ & $4.6 \pm 0.6$ & 0.26 \\
\hline Satisfaction with clerkship evaluation process & $4.2 \pm 0.7$ & $4.4 \pm 0.6$ & 0.09 \\
\hline Satisfaction with feedback from clerkship preceptors & $3.5 \pm 1.1$ & $4.5 \pm 0.6$ & $<0.001$ \\
\hline Overall satisfaction with clerkship & $4.3 \pm 0.5$ & $4.5 \pm 0.5$ & 0.03 \\
\hline
\end{tabular}

Values are presented as mean \pm standard deviation.

${ }^{*}$ From paired t-test for comparing the differences between two means. 
for the clerkship was $4.6 \pm 0.5$, while that for the item concerning students' understanding of the clerkship's evaluation criteria was $3.6 \pm 1.0$ (Table 4).

As for students' satisfaction, the scores were as follows: attitude of clerkship preceptors, $4.8 \pm 0.5$; educational environment for clerkship in a university hospital, $4.6 \pm 0.6$; clerkship evaluation process, $4.4 \pm 0.6$; feedback from clerkship preceptors, $4.5 \pm 0.6$; and the clinical clerkship in the university hospital (overall satisfaction), $4.5 \pm 0.5$ (Table 4).

Additionally, students had a mean score of $4.2 \pm 0.6$ on the item concerning their confidence in interviewing a first-visit outpatient, and a score of $4.7 \pm 0.5$ on the item concerning their awareness of improved interview skills via video recording and professors' feedback.

\section{Comparison of Clerkship Experiences between Community Hospitals or Clinics and the University Hospital}

We then compared the clerkship experiences in the community hospital or clinic with those in the university hospital. The mean score on the item concerning students' recognition of the differences in characteristics of the family medicine outpatients between the two sites was $3.9 \pm 0.7$. Furthermore, their score for the item concerning recognition of the differences in clerkship experiences between the two sites was $3.7 \pm 0.7$.

As for students' understanding of the clerkship objectives, clerkship evaluation criteria, and principles of primary care, we observed no differences between the clerkship sites $(\mathrm{P}>0.05)$. Both satisfaction with the feedback from preceptors and overall satisfaction with the clerkship were significantly higher in the university hospital clerkship $(\mathrm{P}<0.01)$. We observed no significant differences between clerkship sites in terms of satisfaction with the attitude of teachers, the clerkship environment, or the clerkship evaluation process $(\mathrm{P}>0.05)$ (Table 4).

Students' descriptions of the differences between clerkships in the university hospital and that in the community hospital or clinic indicated that patients in community hospitals or clinics tended to have acute and mild illnesses and come from neighboring local communities, whereas patients in the university hospital tended to have chronic and more serious illnesses.

The students also recognized that in the family medicine department of the university hospital, consultation time tends to be longer, medical practice is done more in accordance with the principles outlined in textbooks, and the cost is considerably higher. In contrast, in the community hospital or clinic, consultation time tends to be shorter and practice is more symptomatic and practical. Additionally, the students perceived a difference in content between the types of clerkships. The largest such difference was that, in community hospitals or clinics, the clerkship was observation-based. In contrast, in the university hospital, students have more opportunities for participating in preinterviews with outpatients.

\section{Comparison of Pre-clerkship Expectations and Post-clerkship Experiences}

The students recognized at pre-clerkship that there were differences in the characteristics of family medicine outpatients between community hospitals or clinics and university hospitals; their post-clerkship recognition of this difference did not change significantly $(\mathrm{P}>0.05)$. The students' self-confidence in interviewing a first-visit patient showed a significant increase after the clerkship $(\mathrm{P}<0.01)$, as did their recognition of the effect of teaching medical interviewing skills through video recording and feedback $(\mathrm{P}<0.01)$. We noted that students' recognition of the need for clerkships in a community hospital or clinic was higher after completion of the clerkship $(\mathrm{P}<0.01)$ (Table 1$)$.

\section{DISCUSSION}

Medical students' overall satisfaction with the family medicine clerkships in the university hospital and a community hospital or clinic were high. Thus, even though the community clerkship took place only one day out of a week (5 days), students recognized it as helpful to their understanding of primary care. Similarly, they gave high ratings to the utility of and need for being installed in community hospitals or clinics during their clerkship. These results were similar to those of previous studies on this topic. ${ }^{12,13)}$

A review of students' post-clerkship responses and descriptions of their positive educational experiences showed that the community clerkship gave them the opportunity to observe and confirm how the principles of family medicine as primary care-which they had learned previously in the classroom-are implemented and reflected in a community hospital or clinic.

Furthermore, students indicated that, through the community clerkship, they could better understand the characteristics of patients visiting a family physician's office and the principles and practices of family medicine. More specifically, they showed that they could effectively acquire patient interview skills and understand the doctor-patient relationship by observing an actual practitioner caring for patients, even when formal feedback was deficient. Additionally, students reported that it was helpful to see the reality of family medicine practice and its management and to learn how to open a clinic. Because the clerkship period in community hospitals or clinics is relatively short, students rarely encountered any actual diseases or use of procedural skills. Nonetheless, the students indicated that they had experienced a variety of patient types.

Leone-Perkins et al. ${ }^{7)}$ reported no significant differences in students' experiences of a four-week family medicine clerkship between a resident-teaching hospital and a community clinic; furthermore overall satisfaction was high for the clerkships in both sites. Additionally, students reported that at both the resident-teaching hospital and community clinic, they were given the opportunity to learn the principles of outpatient-based family practice, skills related to comprehensive and continuous primary care, the application of the biopsychosocial approach in clinical practice, and the importance of the doctor-patient 
relationship. The findings in this report, notably, were similar to those of the present study.

In the current survey, the students recognized, at pre-clerkship, that there is a difference in patient characteristics between university hospitals and community hospitals or clinics. Furthermore, this awareness did not change significantly after the clerkship. In the community hospitals or clinics, the students encountered low back pain, osteoarthritis, allergic rhinitis, and immunization more frequently than in the university hospital; however, in the university hospital, they were more likely to encounter depression, anxiety, and headache than in a community hospital or clinic.

As for educational experiences, in the community hospitals or clinics, evidence-based prescription, medical care of the elderly, and practice of clinical procedures were encountered more frequently than in the university hospital. In contrast, family-centered medical care, counseling for smoking cessation, and participation in a journal club were more frequently encountered in the university hospital than in the community hospitals or clinics.

Carney et al. ${ }^{8)}$ compared medical students' clerkship experiences between a university hospital, a community teaching hospital, and community clinics. Their results indicated that experience of clinical procedures was more frequent in community clinics, whereas experience of counseling techniques was more frequent in the university and community teaching hospitals.

In the pre- and post-clerkship comparison, students' expectations about their experiences in community hospitals or clinics, which were already high at pre-clerkship, were unchanged at post-clerkship. For both types of clerkships, students' understanding of the learning objectives of the clerkships was low, as was their understanding of the clerkship evaluation criteria. In particular, in the community hospitals or clinics, there was no feedback from tutors at the end of the clerkship.

Students' self-confidence in performing medical interviews with outpatients was significantly increased after completing the family medicine clerkship, which is likely a direct result of the clerkship's teaching of interview skills. In the post-clerkship questionnaire survey at the university hospital, the students most frequently mentioned that their most valuable educational experience was the pre-interviewing of a first-visit patient and the receipt of feedback from the professor about the video-recorded encounter. Additionally, the students indicated that participating in the professor's outpatient care and directly observing patients with a variety of diseases were positive experiences.

This study has two limitations. First, it was not possible to compare the sociodemographic characteristics of the patients who visited the department of family medicine of the university hospital and those who visited the community hospitals or clinics. Second, the details of the clinical clerkship experiences of the students were not directly collected in the examination rooms during the clerkships themselves; in- stead, they were collected retrospectively, immediately after their clerkship and according to their memory. The study was performed according to the guidelines of the Helsinki Declaration and underwent scrutiny of the institutional review board of Dankook University Hospital.

\section{CONFLICT OF INTEREST}

No potential conflict of interest relevant to this article was reported.

\section{REFERENCES}

1. Choi CJ, Kim BT, Oh SW, Won CW, Yoo SM, Park H, et al. The survey of family medicine clinical clerkships in medical schools in Korea. J Korean Acad Fam Med 2004;25:895-900.

2. Kim JB, Lee Y, Joo EK, Chun KH. Community-oriented primary care: preparing physicians for the future in the United States. Korean J Med Educ 2000;12:329-341.

3. Puvanendran R, Vasanwala FF, Kamei RK, Hock LK, Lie DA. What do medical students learn when they follow patients from hospital to community?: a longitudinal qualitative study. Med Educ Online 2012;17. http://dx.doi.org/10.3402/meo.v17i0.18899.

4. Park YC. Survey of predoctoral education of Korean medical schools: clinical clerkship. Proceedings of the Fall Conference of the Korean Academy of Family Medicine; 2013 Oct 11-13; Seoul, Korea. Seoul: Korean Academy of Family Medicine; 2013.

5. Kim BT, Shin DH. Clinical clerkship in a community clinic. J Korean Acad Fam Med 2004;25:S394-8.

6. Greer T, Schneeweiss R, Baldwin LM. A comparison of student clerkship experiences in community practices and residency-based clinics. Fam Med 1993;25:322-6.

7. Leone-Perkins M, Schnuth RL, Lipsky MS. Students' evaluations of teaching and learning experiences at community- and residencybased practices. Fam Med 1999;31:572-7.

8. Carney PA, Eliassen MS, Pipas CF, Genereaux SH, Nierenberg DW. Ambulatory care education: how do academic medical centers, affiliated residency teaching sites, and community-based practices compare? Acad Med 2004;79:69-77.

9. Prunuske JP, Deci DM. Learning environment: the impact of clerkship location on instructional quality. Fam Med 2013;45:193-6.

10. Masters PA, Nester C. A study of primary care teaching comparing academic and community-based settings. J Gen Intern Med 2001;16:913.

11. Malloy MH, Speer A. A comparison of performance between thirdyear students completing a pediatric ambulatory rotation on campus vs in the community. Arch Pediatr Adolesc Med 1998;152:397-401.

12. Lee J, Park HK. Evaluation of community primary care clerkship. J Korean Acad Fam Med 1999;20:812-21.

13. Okayama M, Kajii E. Does community-based education increase students' motivation to practice community health care?: a cross sectional study. BMC Med Educ 2011;11:19. 